\title{
Piloting a System for Behavioral Surveillance Among Heterosexuals at Increased Risk of HIV in the United States
}

\author{
Elizabeth A. DiNenno*, Alexandra M. Oster, Catlainn Sionean, Paul Denning and Amy Lansky \\ Behavioral and Clinical Surveillance Branch, Division of HIV/AIDS Prevention, National Center for HIV, Viral \\ Hepatitis, STD, and TB Prevention, Centers for Disease Control and Prevention, USA
}

\begin{abstract}
Objectives: During the past decade, the number and proportion of reported HIV cases in the United States acquired through heterosexual contact has increased markedly. CDC employs the National HIV Behavioral Surveillance System (NHBS) to monitor risk behaviors and HIV prevalence in high-risk populations. To identify a target population for conducting NHBS among heterosexuals at increased risk for HIV (NHBS-HET), CDC designed, implemented and evaluated a pilot study.

Methods: The pilot study was conducted in 25 US metropolitan statistical areas in 2006-7. We recruited men and women who reported sex with at least one opposite-sex partner during the past year for a behavioral survey and HIV test. We investigated the relationship between newly diagnosed HIV infection and individual risk behaviors, sexual network characteristics, and social-structural characteristics to arrive at a definition of a heterosexual at increased risk of HIV.

Results: Of 14,750 participants in the analysis, 207 (1.4\%) had newly diagnosed HIV infection. Using low socioeconomic status (SES) as a criterion for defining a heterosexual at increased risk for HIV resulted in optimal rates of HIV prevalence, specificity, sensitivity and practicality.

Conclusions: Results from the NHBS pilot study underscore the key role of social factors as determinants of HIV infection risk among U.S. heterosexuals, and low SES was incorporated into the definition of a heterosexual at increased risk for HIV in NHBS-HET cycles. Future cycles of NHBS-HET will help tailor prevention programs for those populations most at risk of HIV in the US.
\end{abstract}

Keywords: Behavioral surveillance, heterosexual at-risk, HIV, low socioeconomic status, poverty, social determinants of health.

\section{BACKGROUND}

In the United States, the proportion of HIV diagnoses attributed to heterosexual contact rose from approximately $12 \%$ of all cases reported to CDC in 1995 to $31 \%$, or 12,860 cases out of 42,011 reported, in 2009; heterosexual contact now accounts for more newly diagnosed cases of HIV and AIDS than injection drug use [1].

CDC conducts surveillance to monitor the occurrence of HIV in the U.S. population. However, given that HIV infection may be asymptomatic for years, behaviors reported at the time of diagnosis may not reflect behaviors at the time of acquisition. Therefore, it is important to identify populations that are at increased risk for HIV infection and collect information on their behaviors to implement and evaluate effective public health actions [2].

In 2003, CDC implemented the National HIV Behavioral Surveillance System (NHBS), which conducts surveillance of behavioral risks and HIV prevalence in metropolitan statistical areas (MSAs) with high AIDS prevalence among 3 populations at high risk for acquiring HIV: men who have sex with men (MSM), injecting drug users (IDU), and

*Address correspondence to this author at the Behavioral and Clinical Surveillance Branch, Division of HIV/AIDS Prevention, CDC, 1600 Clifton Road (MS E-46), Atlanta, GA 30333, USA; Tel: 404639 8482;

E-mail: Eid8@cdc.gov heterosexuals. To develop NHBS, definitions of the target populations as well as sampling methods were required. Persons who engage in male-male sexual behavior or injection drug use substantially increase their HIV acquisition risk. For instance, in a recent survey of the general U.S. population, approximately $10 \%$ of adult men who report lifetime sexual contact with another man tested positive for HIV [3]. In contrast, prevalence of HIV among heterosexuals who do not inject drugs or engage in malemale sex in the US is very low $(0.1-0.2 \%)$ [4].

For heterosexuals, defining the target population is less clear than that of MSM or IDU. Because HIV prevalence is so low in the general population, sex with an opposite sex partner is not a good marker for HIV acquisition risk. The National HIV Surveillance System attributes cases to heterosexual contact if a person reports sexual contact with a person known to have or be at high risk for HIV (e.g., a partner who injects drugs); however, information about partners' risk behaviors is not always known, making this an inadequate definition for purposes of a behavioral survey. In other countries, behavioral surveillance focuses on specific groups of heterosexuals such as commercial sex workers and their clients [5] or those migrating from high prevalence areas [6]. Another challenge is determining scientifically sound, feasible, and replicable strategies for sampling heterosexuals at increased risk for surveillance purposes. Behavioral surveys among MSM have shown venue-based 
sampling (VBS) to be an effective way to reach men at risk; similarly, respondent-driven sampling (RDS) has been used successfully to reach IDU. These methods were used to recruit MSM in NHBS (NHBS-MSM) and IDU in NHBS (NHBS-IDU), respectively [7,8].

This paper describes the design, implementation, and evaluation of a pilot study to conduct community-based behavioral surveillance among heterosexuals at increased risk for HIV (NHBS-HET) in the United States, and how the findings from the pilot study were applied to subsequent NHBS-HET surveillance activities.

\section{METHODS}

\section{NHBS-HET Pilot Study Development}

In spring 2005, CDC convened a series of internet-based webinar consultations with experts in the field of heterosexually acquired HIV infection in an effort to begin designing the NHBS-HET pilot study. The purpose of the consultations was to assist CDC define the target population (heterosexuals at increased risk for HIV infection) that would be used in the pilot study. The structure for the discussions was based on a systematic review of the literature conducted by $\mathrm{CDC}$ which had identified three major themes about determinants of HIV risk for heterosexually-active persons: individual risk behaviors (behaviors that place individuals at higher risk of HIV); sexual networks (characteristics of relationships between sex partners that increase HIV risk); and social/structural context (population characteristics that place persons in communities at higher risk of HIV). Based on this framework from the literature review and the discussions during the webinars, the consultants made several recommendations regarding eligibility criteria for the pilot study. They recommended against using individual risk behaviors, such as having multiple partners, for eligibility because that would exclude persons who do not engage in high-risk behavior. They also recommended against using the HIV status or risk behaviors of partners as eligibility criteria because many people may not know this information about their partners. The consultants did recommend using "high risk areas," defined as geographic areas within the NHBS MSAs with high rates of heterosexually acquired HIV, sexually transmitted diseases (STDs), poverty, and incarceration, as eligibility criteria because these serve as a proxy for risk within the sexual network as well as a measure of social/structural context.

Based on these recommendations, methods were developed to identify high risk areas (HRAs) that could be used by all of the NHBS project sites. Because comparable datasets could not be identified for STD rates and incarceration rates for all NHBS MSAs, HRAs were ultimately defined as census tracts within NHBS MSAs that had high rates of HIV/AIDS diagnoses attributed to heterosexual contact and high rates of poverty. An index combining these two measures was created, index values were plotted and a cutoff was determined using scree plots to identify HRAs in MSAs. Typically, $<20 \%$ of census tracts within MSAs were classified as HRAs.

Although not specified by the consultants, an upper age limit was added to the eligibility criteria to ensure that persons in age groups most at risk for HIV infection were included. Because persons aged 50 and older account for only a relatively small proportion of all HIV diagnoses in the United States [1], the upper age limit for eligibility for NHBS-HET was set at 50 years. Consistent with other NHBS cycles, the lower age limit was 18 [9].

Four potential sampling methods were identified for use in the NHBS-HET pilot study: population-based household sampling, adaptive sampling (a procedure for selecting people to be in the sample that depends on variables of interest observed during the survey, so the design adapts to the population as encountered) [10], respondent-driven sampling (RDS), and venue-based, time-space sampling (VBS). Advantages and disadvantages of each sampling strategy were presented to NHBS principal investigators for discussion and joint decision making. While household and adaptive sampling could provide unbiased estimates, they were considered by NHBS investigators to be difficult to operationalize. In contrast, the challenges and benefits of RDS and VBS were well known to the NHBS investigators as a result of the previous MSM and IDU cycles. These two methods were the final choices for the NHBS-HET pilot study.

\section{NHBS-HET Pilot Study Implementation}

The NHBS-HET pilot study was conducted in the following 25 MSAs : Atlanta, GA; Baltimore, MD; Boston, MA; Chicago, IL; Dallas, TX; Denver, CO; Detroit, MI; Fort Lauderdale, FL; Houston, TX; Las Vegas, NV; Los Angeles, CA; Miami, FL; New Haven, CT; New Orleans, LA; New York, NY; Nassau-Suffolk, NY; Newark, NJ; Norfolk, VA ; Philadelphia, PA; San Diego, CA; San Francisco, CA; San Juan, Puerto Rico; Seattle, WA; St. Louis, MO; and Washington, DC (Fig. 1). These metropolitan areas represented approximately $60 \%$ of all AIDS cases in urban areas in 2006. Fifteen sites were assigned to recruit participants using RDS and 10 using VBS. (Data from Norfolk, VA were corrupted during data transfer to CDC, which resulted in data from 24 final MSAs for the final analysis) (Fig. 1).

NHBS-HET RDS procedures were similar to those used for the NHBS-IDU cycle [8]. Briefly, recruitment chains began in each MSA with 5-10 initial participants who either were referred from programs serving the local community or were recruited by NHBS staff members through outreach. Initial participants who completed the interview were asked to recruit three other persons through the use of a coded coupon system that tracked the referrals. Recruitment continued for multiple waves of peer referrals. Only participants who lived in an HRA were eligible to recruit peers.

Project sites using VBS first identified a limited number (5-10) of HRAs with the highest index scores to recruit participants. These HRAs were selected to maximize the reach of the sample across different parts of the MSA. VBS activities such as creating a venue universe, narrowing that list to a venue sampling frame, and identifying venuespecific day-time periods were conducted for NHBS-HET in a manner similar to the NHBS-MSM protocol [7]. Venues included retail businesses (e.g., laundromats, beauty salons, pawn shops, grocery and liquor stores), bars, restaurants, 


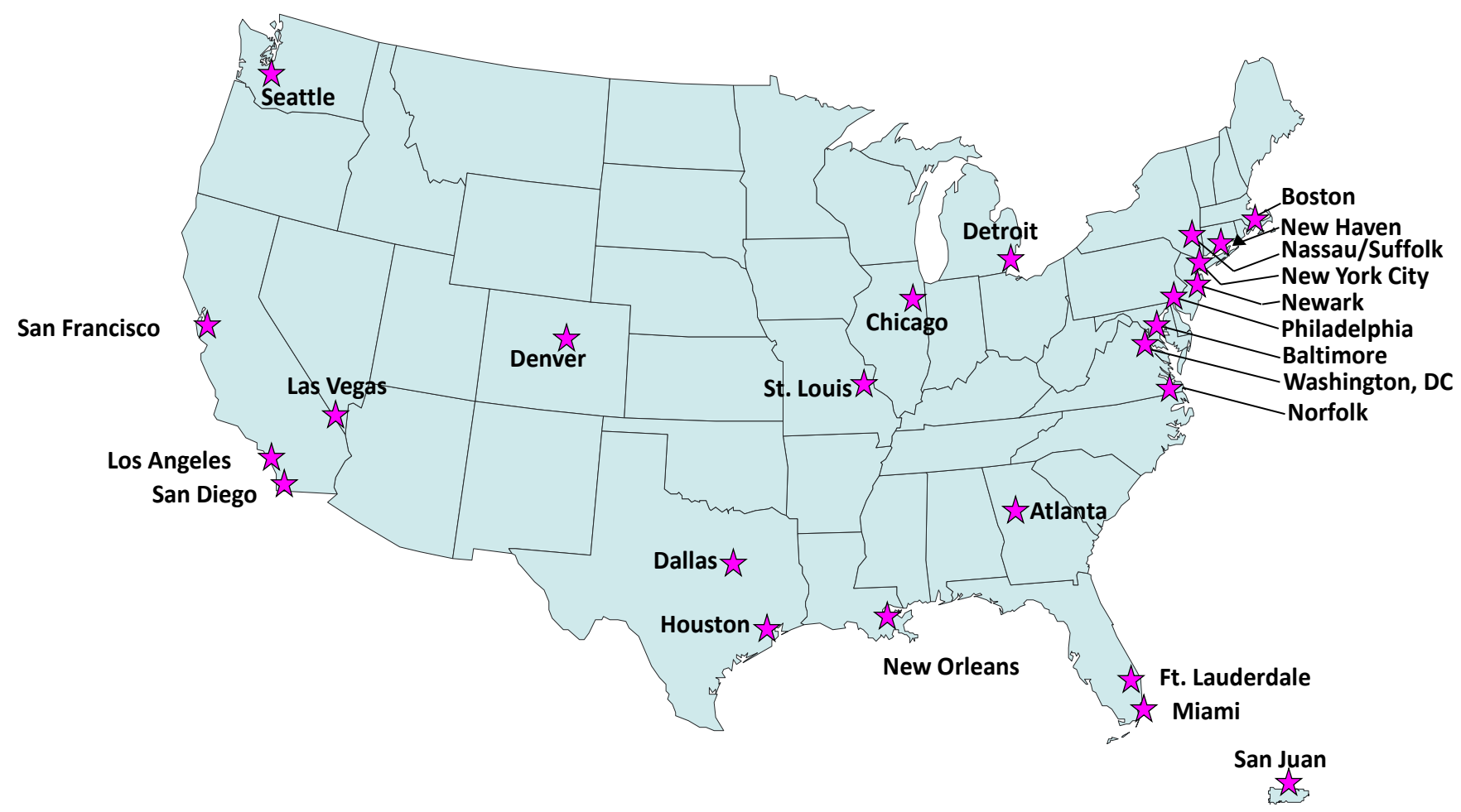

Fig. (1). National HIV Behavioral Surveillance (NHBS) System, Heterosexuals at-risk Pilot study, 2006-2007.

social and religious organizations, sex strolls, high-traffic street locations, parks, and special events such as festivals and block parties. During the study period, venues and the corresponding day/time periods were chosen randomly each month. Staff members then systematically approached men and women at the venues. All venues were located within HRAs.

An eligibility screener was administered to all potential participants. Persons were eligible for the NHBS-HET pilot study if they were a male or female aged 18-50 years, had sex with at least one opposite-sex partner in the past year, lived in the participating MSA, were able to complete the interview in English or Spanish, and had not previously participated in the current cycle of NHBS. After eligible participants gave informed consent, trained interviewers administered a standardized, anonymous questionnaire using a handheld computer. The interview consisted of questions about sex, drug use, HIV testing behaviors, and use of HIV prevention services. The recall period for most questions was the past 12 months.

All respondents were offered anonymous HIV testing (regardless of self-reported HIV infection status), given the opportunity to receive their test results, and those testing positive were anonymously referred to care. HIV testing was performed by collecting blood or oral specimens for either conventional laboratory testing or rapid testing in the field. A non-reactive rapid test was considered negative. For reactive rapid test results, final positive test results were determined based on confirmatory Western blot or immunofluorescence assay.

Although the usual target NHBS sample size is 500 completed interviews per MSA, the target sample size for the NHBS-HET pilot study was 750 completed interviews per MSA to ensure adequate ability to conduct analyses for the purposes of evaluation. Participants were compensated both for their time participating in the interview and for taking an HIV test. In project sites using RDS, participants who recruited their peers were paid an additional incentive for each eligible person they recruited to participate.

The NHBS-HET pilot study was considered research, but the CDC Human Research Protections Office determined that $\mathrm{CDC}$ was "not engaged" in this research and therefore the protocol was not reviewed by the CDC IRB. Each project site followed state and local procedures to determine whether NHBS-HET activities required local IRB review, and obtained approvals as necessary.

\section{NHBS-HET Pilot Study Data Analysis}

In an effort to define the target population for future NHBS-HET activities, we assessed eligibility criteria used in the pilot study as well as other variables collected in the survey that had been identified in the literature as associated with heterosexual acquisition of HIV infection. We chose newly diagnosed HIV infection as the outcome measure to identify factors associated with risk for infection. Persons with newly diagnosed HIV infection were participants who had a positive NHBS HIV test result but who reported in the survey that they had never previously tested HIV-positive.

For this analysis, we only included persons who met eligibility requirements for the pilot, completed the survey, had a final HIV test result that was either positive or negative, and did not report previously testing positive for HIV infection. Furthermore, because we were interested in a population at risk of HIV acquisition through heterosexual behavior, we further limited this analysis to men and women who had never injected drugs and excluded men who reported ever having a male sex partner. 
Predictor variables of newly diagnosed HIV infection fell into three categories:

1. Individual behavior risks: crack use, engaging in exchange sex, having multiple sex partners, incarceration, and STD diagnosis;

2. Sexual network risks: reporting that one's last opposite-sex partner was HIV-infected, a man who has sex with men, an injection drug user, a crack user, or incarcerated, or not knowing the HIV status of one's partner; having concurrent sex partners or a partner with concurrent partners; and

3. Social/structural risks: living in a high risk area, having a low household income, and having limited education.

We evaluated each predictor variable or combination of variables as ways of defining the target population using 4 criteria: HIV prevalence must be greater than that of the overall sample prevalence $(>1.4 \%)$, specificity must be relatively low $(<=40 \%)$, sensitivity must be relatively high $(>=70 \%)$, and the variable must be practical to use in a national surveillance system. A high prevalence $(>1.4 \%)$ was necessary in order to screen out populations at lower risk of infection. If a variable satisfied this criterion, then we evaluated sensitivity (the proportion of newly diagnosed, HIV-positive participants who met the criterion) and specificity (the proportion of HIV-negative participants who did not meet the criterion). In contrast to a normal screener, in which a high specificity is generally desirable, we wanted to be sure that we would not screen out a large number of potential participants as on the basis of a particular variable for two main reasons. First, if too many potential participants are not eligible, recruitment can be compromised as the credibility of the project becomes undermined in the community. For a variable with specificity $<40 \%$, less than half of all potential participants would be deemed ineligible, which was determined to be the maximum proportion allowable to not adversely affect overall recruitment. Second, because the purpose of NHBS is to monitor populations at risk of acquiring HIV, we wanted to be sure that we would capture a large population of heterosexuals who were at risk of HIV infection rather than a population who were already infected. Variables that performed well with respect to HIV prevalence, sensitivity, and specificity were assessed for practicality-how feasible and useful a variable was as a potential eligibility screener. We considered whether use of a particular variable would render us unable to monitor important behaviors, or whether the variable was associated with newly diagnosed HIV infection in only some heterosexual sub-populations (e.g., in some regions of the country).

\section{RESULTS}

Of the 18,430 participants in the final NHBS-HET pilot data set who completed an interview, 14,750 (80\%) met our analysis criteria. Participants were excluded from the analysis if they ever reported injection drug use $(\mathrm{N}=2,241)$, had male-male sex $(\mathrm{N}=414)$, both injected drugs and had male-male sex $(\mathrm{N}=311)$, or if they refused to provide this information $(\mathrm{N}=5)$. Participants were also excluded if they did not consent to an HIV test $(\mathrm{N}=377)$, did not have a negative or confirmed positive HIV test result $(\mathrm{N}=212)$ or reported being HIV-positive but, when tested, were HIVnegative $(\mathrm{N}=5)$. Additionally, we excluded persons whose survey responses were invalid $(\mathrm{N}=120)$. Of the participants in the sample, $207(1.4 \%)$ had newly diagnosed HIV infection. Characteristics of participants and those with newly diagnosed HIV infection are presented in Table 1. Of the 14,750 NHBS-Pilot Study participants who met the analysis criteria, $57 \%$ were female, nearly half were less than 30 years old, and most (73\%) were black (Table 1). Socioeconomic status was low. Few participants had a pastyear risk behavior (traditionally) associated with HIV infection such as crack use (11\%), exchange sex for money or drugs $(12 \%)$ or STD diagnosis $(14 \%)$.

Table 1. Sample Characteristics, Pilot Study of National HIV Behavioral Surveillance System among Heterosexuals at Increased Risk, 2006-2007

\begin{tabular}{|c|c|c|c|c|}
\hline \multirow[t]{2}{*}{ Characteristics } & \multicolumn{2}{|c|}{$\begin{array}{c}\text { All } \\
\text { Participants }\end{array}$} & \multirow{2}{*}{$\begin{array}{c}\begin{array}{c}\text { Newly Diagnosed } \\
\text { HIV-Positive } \\
\text { Participants }\end{array} \\
\text { No. }\end{array}$} & \multirow[t]{2}{*}{$\%$} \\
\hline & No. & $\%$ & & \\
\hline \multicolumn{5}{|l|}{ Gender } \\
\hline Male & 6,315 & 43 & 87 & 42 \\
\hline Female & 8,435 & 57 & 120 & 58 \\
\hline \multicolumn{5}{|l|}{ Age Group } \\
\hline $18-29$ years & 7,086 & 48 & 29 & 14 \\
\hline $30-39$ years & 3,418 & 23 & 55 & 27 \\
\hline $40-50$ years & 4,246 & 29 & 123 & 59 \\
\hline \multicolumn{5}{|l|}{ Race/Ethnicity } \\
\hline White, not Hispanic & 790 & 5 & 6 & 3 \\
\hline Black, not Hispanic & 10,699 & 73 & 171 & 83 \\
\hline Hispanic & 2,679 & 18 & 26 & 13 \\
\hline Others* & 573 & 4 & 4 & 2 \\
\hline \multicolumn{5}{|l|}{ U.S. Census Region ${ }^{\dagger}$} \\
\hline Northeast & 4,272 & 29 & 92 & 44 \\
\hline South & 4,172 & 28 & 82 & 40 \\
\hline Midwest & 2,159 & 15 & 16 & 8 \\
\hline West & 3,537 & 24 & 13 & 6 \\
\hline Territories & 610 & 4 & 4 & 2 \\
\hline \multicolumn{5}{|c|}{ Low Socioeconomic Status ${ }^{\S}$} \\
\hline No & 1,734 & 12 & 9 & 4 \\
\hline Yes & 13,015 & 88 & 198 & 96 \\
\hline \multicolumn{5}{|l|}{ HRA Resident** } \\
\hline No & 2,612 & 18 & 27 & 13 \\
\hline Yes & 12,074 & 82 & 180 & 87 \\
\hline Total & 14,750 & 100 & 207 & 100 \\
\hline
\end{tabular}

Includes American Indian/Alaska Natives, Asians, Native Hawaiian or other Pacific Islanders, persons of multiple race, and those for whom race/ethnicity was missing. 'Northeast: Boston, Massachusetts; Nassau/Suffolk counties, New York; New Haven, Connecticut; New York, New York; Newark, New Jersey; and Philadelphia, Pennsylvania. South: Atlanta, Georgia; Baltimore, Maryland; Dallas, Texas; Fort Lauderdale, Florida; Houston, Texas; Miami, Florida; New Orleans, Louisiana; and Washington, DC. Midwest: Chicago, Illinios; Detroit, Michigan; and St. Louis, Missouri. West: Denver, Colorado; Las Vegas, Nevada; Los Angeles, California; San Diego, California; San Francisco, California; and Seattle, Washington. Territories: San Juan, Puerto Rico.

${ }^{\S}$ Participant's household income did not exceed U.S. Health and Human Services (HHS) poverty guidelines (http://aspe.hhs.gov/poverty/07poverty.shtml) or participant had high school education or less.

${ }^{* *}$ Participants whose census tract of residence could not be identified were excluded from this analysis. 
Table 2 shows the performance of the predictor variables, including the number and proportion of participants, HIV prevalence, sensitivity and specificity. For each of the five individual risk behavior variables, HIV prevalence was between $2.1 \%$ and $3.1 \%$. However, because relatively small proportions of participants engaged in these behaviors $(8-21 \%)$, and sensitivity was low for each (range: $13-32 \%$ ), we removed these individual variables from consideration. Evaluation of combined predictor variables, in which persons reporting any or more than one of these five factors would meet the criteria, resulted in improved sensitivity and specificity but did not meet our threshold for further consideration.

Four predictor variables assessed sexual network risk. For each of these variables or combinations of variables, the HIV prevalence exceeded the overall sample prevalence of $1.4 \%$. Two of these variables met sensitivity and specificity criteria for further evaluation: 1) reporting not knowing the HIV status of one's most recent partner or that one's most recent partner was an IDU, had been incarcerated, used crack or, for women, was MSM; and 2) sexual concurrency.
We also present data on several social/structural factors including living in an HRA: low income (participant's household income did not exceed Department of Health and Human Services [HHS] poverty guidelines); and limited education (high school graduate or less). Each of these performed well with respect to HIV prevalence, sensitivity, and specificity. A combined variable including anyone with low income or limited education (which we termed low socioeconomic status [SES]) performed even better, with a sensitivity of $96 \%$ and specificity of $12 \%$.

Practicality was assessed for variables meeting criteria for sensitivity $>70 \%$ and specificity $<40 \%$. Use of sexual network measures as eligibility criteria would limit our ability to monitor partner HIV status and other characteristics over time. The use of these variables is also limited by the concern that a large proportion of people did not know the risk behaviors or HIV status of their partners. Use of questions based on characteristics of the most recent partner also might underestimate risk for persons with more than one partner. As a result of these limitations, sexual

Table 2. Sensitivity and Specificity Performance of Potential Eligibility Criteria by HIV Prevalence, Pilot Study of National HIV Behavioral Surveillance System Among Heterosexuals at Increased Risk, 2006-2007

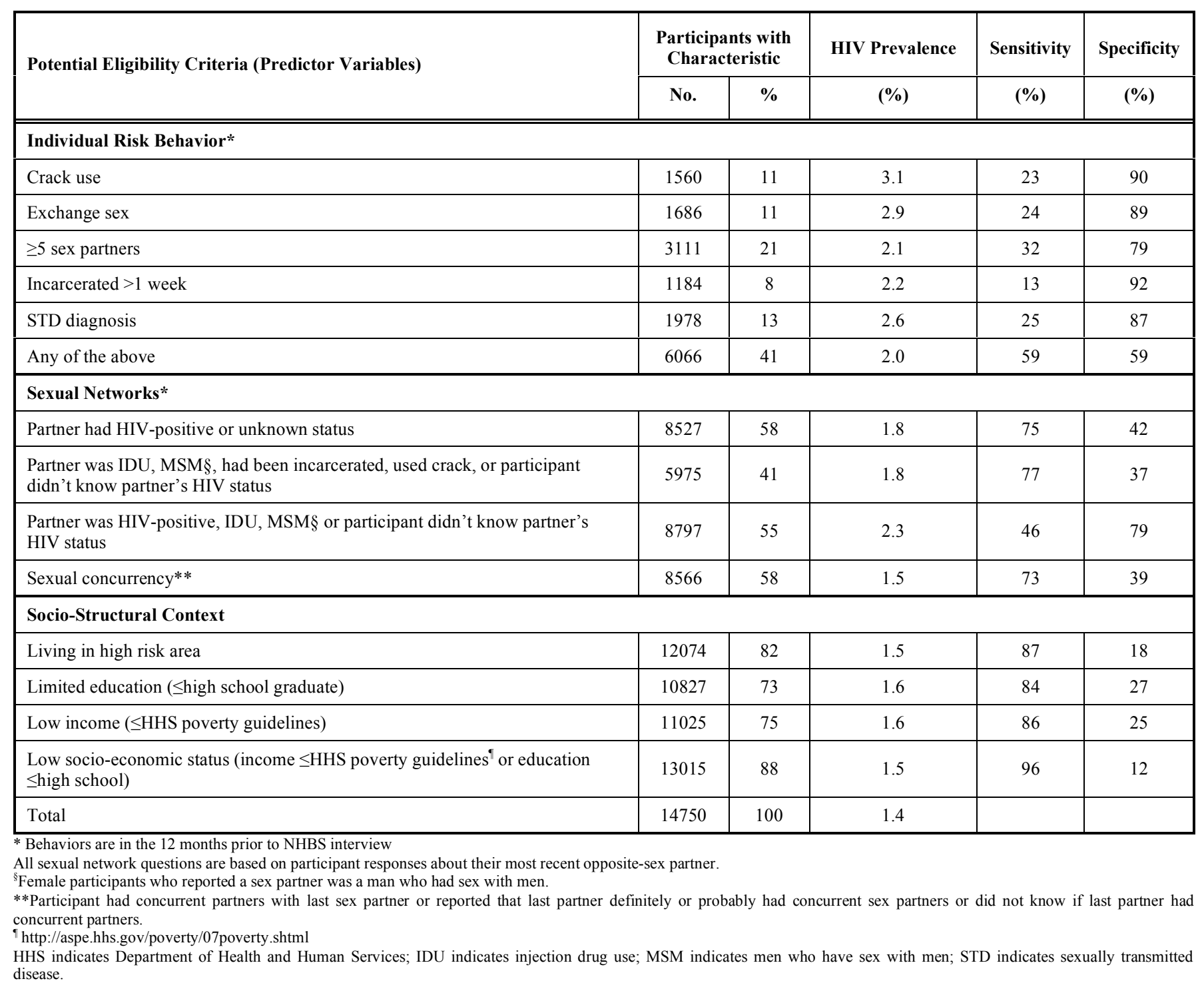


network risks were eliminated from consideration as potential ways to identify the target population eligibility criteria.

All social/structural factors presented met the threshold for further evaluation. Of these, living in an HRA and low SES performed best and were evaluated for practicality. Use of residency in an HRA was less optimal for several reasons. For instance, some participants had difficulty identifying their census tract of residence on a map; because NHBS is an anonymous survey, staff do not collect address or other detailed information that would make identifying census tract of residency easier. Furthermore, when performance of HRA residency as a predictor variable was assessed by region, it was determined that it performed poorly in the Midwest and the West because a smaller proportion of participants lived in HRAs (data not shown), which would have been problematic for a national surveillance system. The HRA criterion also performed worse in project sites that used RDS than those that used VBS; participants with newly diagnosed HIV infection recruited through the RDS method were less likely to live in an HRA than those recruited through the VBS method.

In contrast, the use of low SES as a method for defining the target population posed fewer concerns with respect to practicality. Measurement of self-reported income and education is less complicated than residency in an HRA. Moreover, it had excellent sensitivity in cities within all U.S. regions and territories and among all heterosexual subpopulations (data not shown).

The results of these analyses were presented at an NHBS principal investigators' meeting in October 2009 and representatives from each NHBS project site were asked to weigh the evidence and make a recommendation about 1) the final definition of the target population and its operationalization through specific eligibility criteria, and 2) the sampling method that should be used. RDS was the recommended sampling method for NHBS-HET, endorsed by project areas that had used VBS as well as those that used RDS.

The final NHBS-HET definition incorporated the low SES criteria described above. Participants for NHBS-HET would be eligible if they met the general NHBS eligibility criteria (live in the MSA, not previously participated in current cycle, able to complete the interview in English or Spanish), are male or female, have had vaginal or anal sex with an opposite-sex partner in the previous 12 months, and are aged 18-60 years. The upper age limit was increased to 60 years based on the high prevalence of new diagnosis among those aged 40-50 years in the pilot study. The SES criteria were incorporated into the RDS method by offering only NHBS-HET participants who are low SES as measured by income and education (described above) and have not injected drugs in the previous 12 months an opportunity to recruit others into the study. By using low SES as recruitment criteria, but not as eligibility criteria, we sought to limit the potential inconvenience to participants traveling to a field site only to be found ineligible for the study, and the potential negative impact the study might suffer as a result. However, since only persons meeting the recruitment criteria may recruit others, the resulting sample would be comprised primarily of the low SES target population. The
HRA concept is applied only for initial participants, who must be residents of an HRA; in addition, field sites must be located within HRAs. These HRA criteria make sure that the study starts in, and recruitment is maintained within, lower SES communities. This combination of eligibility and recruitment criteria, in conjunction with the RDS method, were selected to ensure that future cycles of NHBS-HET attain a sample of heterosexuals at increased risk for HIV infection.

\section{DISCUSSION}

The NHBS-HET pilot study resulted in a technique to identify heterosexuals at risk for HIV infection and a method to sample them. Our findings were based on nearly 15,000 interviews conducted among persons in MSAs with high AIDS prevalence across the United States. Following an investigation of the performance of predictor variables for heterosexuals with newly diagnosed HIV infection, we determined that the definition of a heterosexual at increased risk would focus on persons with low SES. Eligibility for future NHBS-HET cycles would be limited to a man or woman living in an NHBS MSA who has had vaginal or anal sex with an opposite-sex partner in the previous 12 months; the opportunity to recruit other participants would be offered only to participants with low socioeconomic status (measured by high poverty or low education) and no injecting-drug use in the previous 12 months. Together, these eligibility and recruitment criteria comprise our definition of heterosexuals at increased risk of HIV.

The pilot study analysis was an important step in the effort to better conceptualize heterosexual risk of HIV. Earlier research on the topic defined heterosexuals at risk solely on the basis of high risk sexual behavior [11], such as sex with multiple partners [12]. Results from the NHBS pilot study shift this focus from risky behaviors to at-risk communities by emphasizing the role of social factors specifically, low SES - in which individuals exist and behaviors occur. Other contemporaneous research highlights the importance of patterns of sexual partnership formation, as well as the social conditions that are associated directly or indirectly with the likelihood of having an infected partner [13].

Our focus on social factors also mirrors other research conducted in the last decade, which has revealed that poverty $[13,14]$ and concomitant social disparities such as racial inequality $[15,16]$ are underlying determinants of HIV infection risk [17] even in people who do not have high-risk behaviors [18]. Differences in sexual behaviors have also been shown to not account for increased risk for STDs among blacks [19, 20], and that STD disparities between blacks and whites are greater among individuals in low-risk groups than those in high risk groups [21]. In addition, a previous analysis of NHBS-HET pilot study data demonstrated that race was not significant in a multivariate model which included SES, suggesting that low SES, not black or Hispanic race/ethnicity, is more appropriate to define a high-risk heterosexual population in the U.S. [22]. Nonetheless, the link between low SES and HIV infection among heterosexuals remains unclear and must be investigated further. A recent study of U.S. women - the Women's HIV SeroIncidence Study (ISIS) - utilized some 
aspects of the NHBS-HET pilot study design to enroll and retain women at risk for HIV infection in a longitudinal HIV incidence study. However, unlike NHBS, ISIS eligibility was based on behavioral risk factors for women such as sex with a partner who is HIV positive, recent injection drug use, and residency in an HRA [23]. Results from ISIS may help clarify the causal link between poverty, risk behaviors, and HIV incidence among heterosexual women in the US.

There are several limitations to our analysis. First, the NHBS-HET pilot study was a convenience sample in which data from two different methods were combined. Data are not weighted to account for biases in venue attendance (VBS) or differences in network size and composition (RDS); therefore, the results are not generalizable to all heterosexuals at risk in the NHBS MSAs or in the United States. Second, NHBS-HET recruitment targeted residents of areas with high rates of poverty and HIV diagnoses, which may have led to an overestimation of HIV prevalence and also may have limited the applicability of our analysis to communities outside of these highly selected areas. Third, positive HIV status may have been underreported during this interviewer-administered survey, thereby inflating estimates of participants with unrecognized infection. The NHBS questionnaire is interviewer-administered which may increase reporting bias especially for sensitive topics such as sex and drug use.

The second cycle of NHBS-HET was conducted from June through December 2010 using the new definition of heterosexual at risk with the eligibility and recruitment criteria derived from the pilot study described in this paper. CDC is currently analyzing results from the 2010 data collection cycle. Future cycles of NHBS-HET, with its focus on people with low SES and the communities they live in, will help tailor and implement prevention programs for those who are most at risk in the effort to stem the tide of heterosexually acquired HIV infection in the United States.

\section{ACKNOWLEDGEMENTS}

This report is based, in part, on contributions by National HIV Behavioral Surveillance system staff members, including: Luke Shouse, Laura Salazar (Atlanta, GA);: Colin Flynn, Frangiscos Sifakis (Baltimore, MD;): Debbie Isenberg, Maura Driscoll, Elizabeth Hurwitz (Boston, MA): Carol Cieselski, Nikhil Prachand, Nanette Benbow (Chicago, IL): Sharon Melville, Richard Yeager, Jim Dyer, Nandita Chaudhuri; Alicia Novoa; (Dallas, TX): Mark Thrun, Alia Al-Tayyib, Ralph Wilmoth (Denver, CO): Renee McCoy, Vivian Griffin, Eve Mokotoff (Detroit, MI): Marcia Wolverton, Jan Risser, Hafeez Rehman (Houston, TX): Deborah McBride, Bob Salcido, Jay DiCotignano, SaBrina Hagan-Finks (Las Vegas, NV): Trista Bingham, Ekow Sey (Los Angeles, CA): Marlene LaLota, Lisa Metsch, David Forrest, Dano Beck, Stefanie White (Miami \& Ft. Lauderdale, FL): Chris Nemeth, Carol-Ann Watson (NassauSuffolk, NY): Aaron Roome, Peg Weeks (New Haven, CT): William Robinson, DeAnn Gruber (New Orleans, LA): Chris Murrill, Samuel Jenness, Holly Hagan, Travis Wendel (New York City, NY): Helene Cross, Barbara Bolden, Sally D'Errico (Newark, NJ): Dena Bensen, Judith Bradford (Norfolk, VA): Kathleen Brady, Althea Kirkland (Philadelphia, PA): Vanessa Miguelino, Al Velasco, Rosana
Scolari (San Diego, CA): Henry Raymond, Willi McFarland (San Francisco CA): Sandra Miranda De León, Yadira Rolón-Colón (San Juan, PR): Maria Courogen, Hanne Thiede, Nadine Snyder, Richard Burt (Seattle, WA): Michael Herbert, Yelena Friedberg, Dean Klinkenberg, LaBraunna Friend (St Louis, MO): Paul Cunningham, Marie Sansone, Tiffany West-Ojo, Manya Magnus, Irene Kuo (Washington, DC).

\section{DISCLAIMER}

The findings and conclusions in this report are those of the authors and do not necessarily represent the views of the Centers for Disease Control and Prevention.

\section{CONFLICT OF INTEREST}

The authors have no institutional or commercial affiliations that might pose a conflict of interest regarding the publication of this manuscript.

\section{REFERENCES}

[1] CDC, 2011.HIV Surveillance Report, 2009; vol. 21. Available at: http://www.cdc.gov/hiv/topics/surveillance/resources/reports/. Published February 2011.

[2] Lansky A, Brooks JT, DiNenno E, Heffelfinger J, Hall HI, Mermin J. Epidemiology of HIV in the United States. J Acq Immun Def Syndr 2010; 55: S64-8.

[3] Xu FJ, Sternberg MR, Markowitz LE. Men who have sex with Men in the United States: demographic and behavioral characteristics and prevalence of HIV and HSV-2 infection results from national health and nutrition examination survey 2001-2006. Sex Transm Dis 2010; 37(6): 399-405.

[4] CDC. HIV prevalence estimates- United States, 2006. MMWR Morb Mortal Wkly Rep 2008; 57: 1073-6.

[5] Weir S, Tate JE, Zhusupov B, Boerma JT. Where the action is: monitoring local trends in sexual behaviour. Sex Transm Infect 2004; 80(Suppl II): ii63-8.

[6] European Centre for Disease Prevention and Control/WHO Regional Office for Europe. HIV/AIDS surveillance in Europe 2010. Stockholm: European Centre for Disease Prevention and Control 2011.

[7] MacKellar DA, Gallagher KM, Finlayson T, Sanchez T, Lansky A, Sullivan PS. Surveillance of HIV risk and prevention behaviors of men who have sex with men - A national application of venuebased, time-space sampling. Public Health Reports 2007; 122: 39-47.

[8] Lansky A, Abdul-Quader AS, Cribbin M et al. Developing an HIV behavioral surveillance system for injecting drug users: The national hiv behavioral surveillance system. Public Health Rep 2007; 122: 48-55.

[9] Gallagher KM, Sullivan PS, Lansky A, Onorato IM. Behavioral surveillance among people at risk for HIV infection in the U.S.: the National HIV Behavioral Surveillance System. Public Health Rep 2007; 122(Suppl 1): 32-8.

[10] Thompson, SK. Adaptive cluster sampling. J Am Stat Assoc 1990; 85: $1050-9$.

[11] Weeks MR, Mosack KE, Abbott M, et al. Microbicide acceptability among high-risk urban U.S. women: experiences and perceptions of sexually transmitted HIV prevention. Sex Transm Dis 2004; 31(11): 682-90.

[12] Dolcini M, Margaret C, Thomas J, Catania JA, Kegeles SM, Hauck WW. Multiple sexual partners and their psychosocial correlates: The population-based AIDS in multiethnic Neighborhoods (AMEN) study. Health Psychol 1995; 14(1): 22-31.

[13] Adimora AA, Schoenbach VJ, Martinson FEA, et al. Heterosexually transmitted HIV infection among African Americans in North Carolina. J Acquir Immune Defic Syndr 2006; 41(5): 616-23.

[14] Holtgrave DR, Crosby RA. Social capital, poverty, and income inequality as predictors of gonorrhea, syphilis, Chlamydia and AIDS cases in the United states. Sex Transm Infect 2003; 79: 62-4, 88 . 
[15] Adimora AA, Schoenbach VJ, Floris-Moore MA. Ending the epidemic of heterosexual HIV transmission among African Americans. Am J Prev Med 2009; 37(5): 468-71.

[16] Aral SO, Adimora AA, Fenton KA. Understanding and responding to disparities in HIV and other sexually transmitted infections in African Americans. Lancet 2008; 372: 337-40.

[17] CDC. Disparities in diagnoses of HIV infection between blacks/African Americans and other racial/ethnic populations-37 states, 2005-2008. MMWR Morb Mortal Wkly Rep 2011; 60(4): 93-8.

[18] Hallfors D, Iritani B, Miller W, Bauer D. Sexual and drug behavior patterns and HIV and STD racial disparities: the need for new directions. Am J Public Health 2007; 97(1): 125-32.

[19] Ellen JM, Aral SO, Madger LS. Do differences in sexual behaviors account for the racial/ethnic differences in adolescent's selfreported history of a sexually transmitted disease? Sex Transm Dis 1998; 25: $125-9$

[20] Harawa NT, Greenland S, Cochran SD, Cunningham WE, Visscher B. Do differences in relationship and partner attributes explain disparities in sexually transmitted disease among young white and black women? J Adolesc Health 2003; 32: 187-91.

[21] McQuillan GM, Kruszon-Moran D, Kottiri BJ, et al. Racial and ethnic differences in the eroprevelance of 6 infectious diseases in the United states: data from NHANES III, 1998-1994. Am J Public Health 2004; 94: 1952-8.

[22] CDC. Characteristics associated with HIV infection among heterosexuals in urban areas with high AIDS prevalence -24 cities, United States, 2006--2007. MMWR Morb Mortal Wkly Rep 2011; 60(31): 1045-9.

[23] The HPTN 064 (ISIS Study) - HIV Incidence in Women at Risk for HIV: US. Hodder S, Justman J, Hughes J, Wang J, Haley D, Adimora A, Del RC, Soto-Torres L, Eshleman S, El-Sadr W, and HIV Prevention Trials Network (HPTN) 064 Study Team. Poster \#1048, Conference on Retroviruses and Opportunistic Infections, 2012; Available at: http://www.retroconference.org/2012b/Abstrac ts/43702.htm [Accessed 3/10/12].

(C) DiNenno et al.; Licensee Bentham Open.

This is an open access article licensed under the terms of the Creative Commons Attribution Non-Commercial License (http://creativecommons.org/licenses/by-nc/ $3.0 /$ ) which permits unrestricted, non-commercial use, distribution and reproduction in any medium, provided the work is properly cited. 